\title{
Significant Liver-Related Morbidity After Bariatric Surgery and Its Reversal-a Case Series
}

\author{
Magdalena Eilenberg $^{1} \cdot$ Felix B. Langer ${ }^{1}$ - Andrea Beer ${ }^{2} \cdot$ Michael Trauner $^{3}$ • \\ Gerhard Prager ${ }^{1} \cdot$ Katharina Staufer $^{4}$ (B)
}

Published online: 30 September 2017

(C) The Author(s) 2017. This article is an open access publication

\begin{abstract}
Background Nonalcoholic fatty liver disease (NAFLD) occurs in up to $80 \%$ of patients with obesity. Current data suggest an improvement of NAFLD after established bariatric procedures.

Objectives This study investigated liver function impairment after Roux-en-Y gastric bypass (RYGB) and one-anastomosis gastric bypass (OAGB).

Setting University Hospital, Bariatric Surgery Unit

Methods In this single-center case series, consecutive in- and outpatients after bariatric surgery who presented with severe liver dysfunction from March 2014 to February 2017 were included and followed until March 2017.

Results In total, 10 patients (m:f $=2: 8$; median age 48 years, range 22-66 years) were included. Liver dysfunction occurred after a median postoperative time of 15 months (range 288 months). Median \%excess weight loss at that time was $110.6 \%$ (range $85.2-155.5 \%$ ). Liver steatosis/fibrosis occurred in $70 \%$, cirrhosis in $30 \%$ of patients, and led to fatigue $(90 \%)$, ascites (70\%), hepatic encephalopathy (30\%), and upper gastrointestinal bleeding (20\%). Elevation of transaminases, impairment of coagulation parameters, thrombocytopenia, and
\end{abstract}

Katharina Staufer

Katharina.Staufer@meduniwien.ac.at

1 Department of Surgery, Division of General Surgery, Medical University of Vienna, Vienna, Austria

2 Department of Pathology, Medical University of Vienna, Vienna, Austria

3 Department of Internal Medicine III, Division of Gastroenterology and Hepatology, Medical University of Vienna, Vienna, Austria

4 Department of Surgery, Division of Transplantation, Medical University of Vienna, Waehringer Guertel 18-20, 1090 Vienna, Austria hypoalbuminemia were present in $70,80,70$, and $100 \%$, respectively. In eight patients, lengthening of the alimentary/ common limb led to an improvement or complete remission of symptoms. In one patient, liver transplantation was required, one patient deceased due to septic shock and decompensated liver disease.

Conclusions Severe liver dysfunction may also occur after bariatric procedures such as OAGB and RYGB. A comprehensive, meticulous follow-up for early identification of postoperative liver impairment should be aspired. Bypass length reduction led to a fast improvement in all patients.

Keywords NAFLD $\cdot$ Weight loss $\cdot$ Liver dysfunction · Bypass reversal

\section{Introduction}

Obesity and the concomitant metabolic syndrome represent a global health care issue. In up to $80 \%$, obesity is associated with nonalcoholic fatty liver disease (NAFLD) including nonalcoholic steatohepatitis (NASH), which may progress to significant liver fibrosis, cirrhosis, and hepatocellular carcinoma [1]. Although lifestyle modification and weight loss is known to be the most effective treatment of NAFLD, long-term reduction of excessive overweight needs discipline and endurance, and is often unsuccessful.

During the last years, bariatric surgery has become an established procedure for effective and sustainable weight loss that may reverse or prevent long-term sequelae associated with obesity, such as cardiovascular disease, diabetes, musculoskeletal damage, and sleep apnea [2]. Importantly, in the majority of patients, bariatric surgery improves liver steatosis, inflammation, and fibrosis in NAFLD patients with obesity [3]. In up to $16 \%$ of cases, yet, an increase of liver fibrosis or 
de novo fibrosis is seen, but is consistently mild without development of cirrhosis or liver function alterations [4-10]. However, occasional deterioration of liver function was reported and may be attributed to the type of bariatric procedure and the extent of malnutrition and malabsorption. Jejunoileal bypass (JIB), and to a lesser extent also biliopancreatic diversion (BPD), are associated with a higher morbidity and mortality, particularly concerning liver function $[11,12]$. Acute liver failure was reported in $7 \%$ in a study following 453 patients after JIB [13]. Therefore, nowadays, bariatric surgeons refrain from performing JIB.

To date, Roux-en-Y gastric bypass (RYGB) and sleeve gastrectomy (SG) are the most commonly performed procedures. The one-anastomosis gastric bypass (OAGB) or omega loop/ mini gastric bypass is an up-and-coming procedure and is regarded as a technically simpler bypass accompanied by an increased \%excess weight loss (\%EWL), but very little complications $[14,15]$. Clinical liver deterioration or histologic liver alterations after OAGB, SG, or gastric banding have not been reported so far.

In the present study, we aimed at highlighting the occurrence and clinical characteristics of liver dysfunction after RYGB, OAGB, and distal GB (performed due to failed weight loss or weight regain) as seen at our institution in a case series of 10 patients.

\section{Patients and Methods}

Consecutive in- and outpatients after bariatric surgery who attended our facility at first presentation of liver dysfunction from March 2014 to February 2017 were included in the study and followed until April 2017. Patients' health records were retrospectively screened for preoperative signs of liver disease and other comorbidities. All available pre- and postoperative results of liver imaging and liver histology, as well as the course of laboratory parameters and clinical symptoms as direct or indirect signs of liver dysfunction were analyzed. NAFLD was graded according to the NAFLD Activity Score (NAS). Additionally, clinical parameters such as age, sex, weight, body mass index (BMI), $\triangle \mathrm{BMI}, \% \mathrm{EWL}$, and \% total weight loss (\%TWL) [16] were documented prior to and/ or after bariatric surgery, respectively. Obesity was graded according to the World Health Organization (WHO) classification [17]. The study was performed in accordance with the Declaration of Helsinki including current revisions.

\section{Results}

\section{Demographics and Initial Body Weight}

Patient characteristics are displayed in Table 1 . Nine of 10 patients suffered from morbid obesity; one patient was classified as having obesity grade II according to the WHO classification [17]. No regular alcohol consumption or other liver diseases than NAFLD were present in any of the patients during the observed time period.

\section{Surgical Procedures and Weight Loss}

In six of 10 patients (\#1-5 and \#8) surgery was performed at our center, whereas patients \#6, 7, 9, and 10 were primarily operated elsewhere and transferred to our facilities only after complications had occurred. RYGB was the most frequently applied primary surgical procedure $(n=5)$. In three patients, OAGB and in two further patients gastric banding was performed (Table 2).

In one patient (\#9) no successful weight loss was achieved after gastric banding $(6.5 \% \mathrm{EWL})$, so that after band removal an OAGB was performed. In the further nine patients, the primary surgical procedure led to a significant weight reduction of $111.8 \%$ median \%EWL (range 75.4-155.5\%; median BMI $22.7 \mathrm{~kg} / \mathrm{m}^{2}$, range $18.4-31.9 \mathrm{~kg} / \mathrm{m}^{2}$; for $\Delta \mathrm{BMI}$ and $\%$ TWL (\%), see Table 2). However, in three of these patients (\#2, 4, and 5), a weight regain occurred after a median time of 7 years (range 1-8 years), in one patient after removal of gastric band due to dysphagia and in two patients after RYGB. Therefore, a conversion to OAGB in the first patient and a shortening of the common channel for reduction of the absorption length in the two other patients were performed (after exclusion of preexisting food restriction). In total, four of 10 patients required a secondary procedure for persistent weight reduction.

Including all primary and secondary surgical procedures, the overall patient's lowest median BMI was $22.2 \mathrm{~kg} / \mathrm{m}^{2}$ (range $18.4-30.5 \mathrm{~kg} / \mathrm{m}^{2}$ ) which was equivalent to a median $\%$ EWL of $110.6 \%(85.2-155.5 \%$, and median $\%$ TWL of $51.6 \%$, range $42.0-61.7 \%$ ). The median remaining intestinal resorption length (alimentary plus common limb) was $357.5 \mathrm{~cm}$ (range 245-500 cm).

\section{Liver Alterations and Course of Liver Deterioration}

The features of liver alterations and clinical presentation are displayed in Tables 1 and 2, as well as Fig. 1. Liver dysfunction developed after a median interval of 15 months (range 288 months), after performing OAGB in four patients (\#2, 3, 8, and 10), after RYGB in two patients (\#6 and 7), and after distal GB following RYGB in two patients (\#4 and 5). In the remaining two patients, liver dysfunction occurred after a symptomfree period of weight stability (84 months after RYGB in patient \#1 and 36 months after OAGB in patient \#9) followed by a further significant weight loss of unknown origin. Four and 5 months thereafter, signs of malnutrition and liver dysfunction became evident (Table 2). 
Table 1 Comparison of laboratory parameters prior to bariatric surgery and thereafter at the onset time of liver function deterioration

\begin{tabular}{|c|c|c|}
\hline $\begin{array}{l}\text { Study population, } \\
n=10\end{array}$ & $\begin{array}{l}\text { Before bariatric } \\
\text { surgery }\end{array}$ & $\begin{array}{l}\text { Peak of liver } \\
\text { dysfunction }\end{array}$ \\
\hline Female sex, \% (n) & $80(8 / 10)$ & $80(8 / 10)$ \\
\hline Age in years, median (range) & $40(21-66)$ & $48(22-66)$ \\
\hline BMI $\left(\mathrm{kg} / \mathrm{m}^{2}\right)$, median (range) & $49.2(38-64)$ & $22.2(20.8-30.5)$ \\
\hline \multicolumn{3}{|l|}{ NAFLD } \\
\hline Liver disease, $\%(n)$ & $60(6 / 10)$ & $100(10 / 10)$ \\
\hline Steatosis/fibrosis, \% (n) & $50(5 / 10)$ & $70(7 / 10)$ \\
\hline Cirrhosis, \% (n) & $10(1 / 10)$ & $30(3 / 10)$ \\
\hline No liver disease & $30(3 / 10)$ & $0(0 / 10)$ \\
\hline Missing, \% (n) & $10(1 / 10)$ & $0(0 / 10)$ \\
\hline \multicolumn{3}{|l|}{ Liver enzymes } \\
\hline AST (U/L), median (range) & $25(14.5-57)$ & $32.5(14-258)$ \\
\hline ALT (U/L), median (range) & $38(12.5-127)$ & $26(11-230)$ \\
\hline GGT (U/L), median (range) & $33(12-110)$ & $38.5(11-468)$ \\
\hline Hypercholesterolemia, \% ( $n)$ & $20(2)$ & $0(0)$ \\
\hline Cholesterol (mg/dl), median (range) & $175.3(109.5-228)$ & $98.5(66-146)$ \\
\hline HDL (mg/dl), median (range) & $52.5(42-59)$ & $42(4-54)$ \\
\hline LDL (mg/dl), median (range) & $111.2(92.1-152.8)$ & $37.2(11.2-92.2)$ \\
\hline Hypertriglyceridemia, \% (n) & $20(2)$ & $10(1)$ \\
\hline Triglycerides (mg/dl), median (range) & $92(46-152)$ & $50.5(28-249)$ \\
\hline T2DM, \% (n) & $10(1)$ & $0(0)$ \\
\hline $\mathrm{OAD}, \%(n)$ & $10(1)$ & $0(0)$ \\
\hline Insulin, \% (n) & $0(0)$ & $0(0)$ \\
\hline HbA1c (\%), median (range) & $5.2(4.7-5.7)$ & $3.9(3.7-4)$ \\
\hline aHTN, \% (n) & $40 \%(4)$ & $10(1)$ \\
\hline Hyperuricemia, \% (n) & $20 \%(2)$ & $0(0)$ \\
\hline Obstructive sleep apnea, \% (n) & $0(0)$ & $0(0)$ \\
\hline $\mathrm{CAD}, \%(n)$ & $0(0)$ & $0(0)$ \\
\hline Congestive heart failure, $\%(n)$ & $10(1)$ & $10(1)$ \\
\hline $\mathrm{PH}, \%(n)$ & $10(1)$ & $10(1)$ \\
\hline
\end{tabular}

$B M I$ body mass index, NAFLD nonalcoholic fatty liver disease, $A S T$ alanin-aminotransferase, GGT gammaglutamyl-transferase, $H D L$ high-density lipoproteins, $L D L$ low-density lipoprotein, $T 2 D M$ type 2 diabetes mellitus, $O A D$ oral antidiabetics, $a H T N$ arterial hyptertension, $C A D$ coronary artery disease, $P H$ pulmonary hypertension
Liver dysfunction was mainly characterized by a moderate increase in liver enzymes (70\%), hepatosplenomegaly $(80 \%)$, thrombocytopenia (70\%), impaired coagulation parameters (80\%), hypoalbuminemia (100\%), and sarcopenia (60\%). At later stages, patients suffered from ascites (70\%), pleural effusions $(30 \%)$, hepatic encephalopathy (30\%), hepatorenal syndrome (10\%), and upper gastrointestinal bleeding (20\%).

The referring histologic picture of liver alterations after bariatric surgery was available in seven patients. While there was a 90 to $100 \%$ micro- and macrovesicular steatosis in two patients (\#1 and 9), in three others a cirrhotic remodeling was predominant (\#2, 3, and 8). In patient \#7 and \#10, a worsening from no comprehensible pathology to macrovesicular steatosis (\#7:20\%; \#10:85\%) and fibrosis (\#7:grade 1a, NAS 3/8; \#10:grade 3; NAS 7/8) was documented. In the other three patients' (\#4, 5, and 6) sonography and/or computed tomography showed steatosis and hepatosplenomegaly, as well as ascites (\#6).

As a consequence, after bridging by implanting a feeding tube into the remnant stomach to increase absorption (\#1, 4 and 7), the common channel was lengthened (at the expense of the biliopancreatic limb) in seven patients (\#3-5, and \#7-10) and the entire bypass reversed in one patient (\#1). In patient \#2, ultimately, liver transplantation was required. Patient \#6 was treated conservatively and died due to septic shock and decompensated liver disease prior to any surgical conversion could have been performed.

Of note, after the original intestinal anatomy was restored in patient \#1, a clinical stabilization occurred as assessed by laboratory parameters (Fig. 1, 1A-E). Furthermore, a 


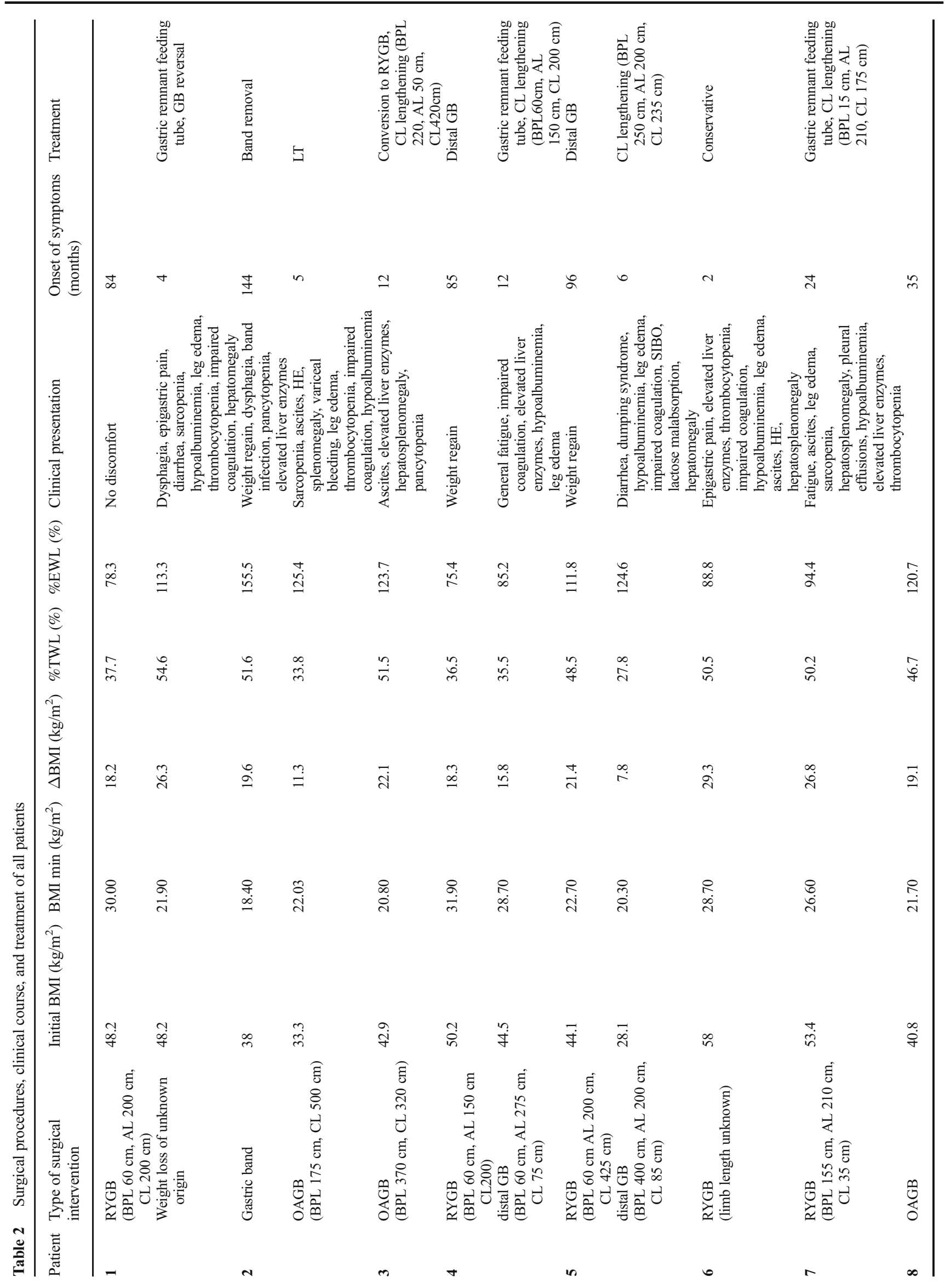




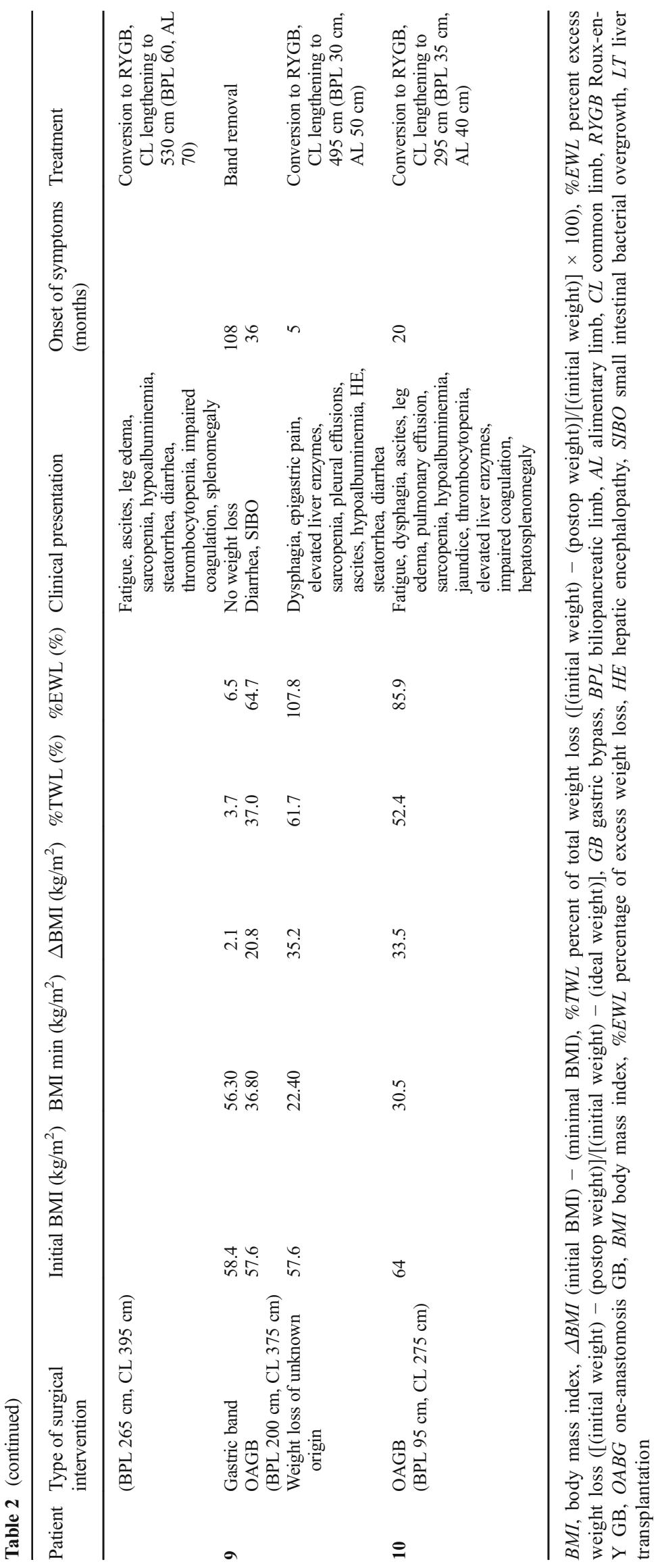



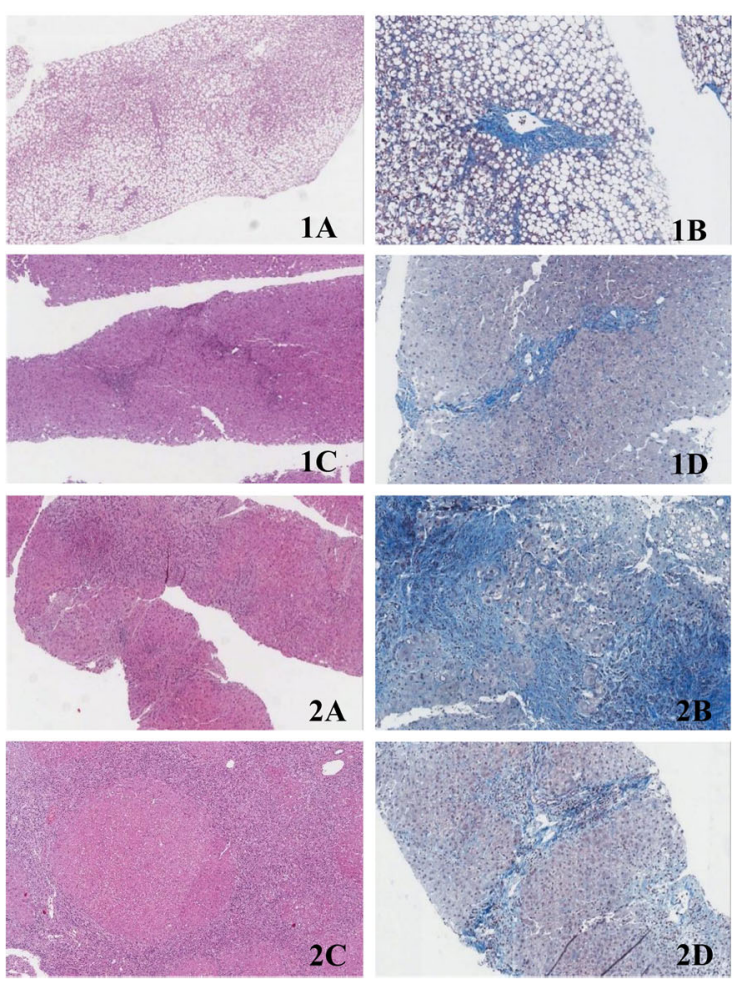

Fig. 1 Histopathological findings of liver biopsy and the corresponding clinical course of patient 1 and 2. Patient \#1 (1A-E): 1A (hematoxylineosin [HE] staining, $\times 8$ magnification [mag.]) and 1B (chromotropaniline blue $[\mathrm{CAB}]$ staining, $\times 20 \mathrm{mag}$.): histology gained at time of feeding-tube implantation; steatosis (95\% macrovesicular, $5 \%$ microvesicular) with partly periportal fibrosis und minor inflammatory activity, correlating with NASH, NA-Score: 3-1-1 (5/8), fibrosis grade 1C. $1 \mathrm{C}$ (HE staining, $\times 10$ mag.), and $1 \mathrm{D}(\mathrm{CAB}$ staining $\times 20$ mag.): histology gained 5 months after bypass reversal: portal and periportal fibrosis, incipient portoportal septation. No micro- or macrovesicular steatosis, no inflammatory activity, no hepatocellular ballooning. 1E: laboratory-values and clinical events over time. Patient \#2 (2A-E): 2A

complete reversal of a prior $100 \%$ steatosis was found and documented by liver biopsy (fig. 1, 1D). In patients \#3 to 5 and \#7 to 10, the lengthening of the common channel at expense of the biliopancreatic limb led to a clinical stabilization and significant improvement both in imaging and laboratory parameters in all patients (data not shown). In patient \#2 (Fig. 1, 2A-E) who received a liver transplant only 11 months after liver transplantation again, transaminases increased to six times the upper limit of normal. Performing a re-conversion operation was considered too risky in view of a common limb of $500 \mathrm{~cm}$ and a questionable benefit of the reversal since liver biopsy additionally revealed de novo post-transplantation autoimmune hepatitis (Fig. 1, 2D).

\section{Discussion}

In the present study, we report 10 cases of significant liver dysfunction after OAGB, RYGB, and distal GB for weight
(HE staining, $\times 8$ mag.): histology gained after explantation of the gastric band: liver tissue with broad, septal fibrosis and starting, focal cirrhotic alteration, no inflammatory activity, corresponding to resolved NASH. 2B (CAB staining, $\times 20$ mag.): histology gained after OAGB: focal cirrhosis, pericellular fibrosis, and moderate steatosis (20\% micro-, 5\% macrovesicular). $2 \mathrm{C}$ (HE staining, $\times 8$ mag.): histology gained at time of liver transplantation (LT): liver cirrhosis and siderosis, marginal irregular steatosis (10\% microvesicular), Ludwig-Score: portal: 2, lobular: 1, fibrosis grade 4 . $2 \mathrm{D}$ (CAB staining, $\times 20$ mag.): histology gained 4 months after LT: hepatic picture with minor inflammatory activity, cholestasis, and focal portoportal fibrosis. 2E: laboratory values and clinical events over time

loss failure or weight regain following RYGB. Liver dysfunction occurred after a median time period of 15 months after surgery had been performed. In two of 10 patients, liver dysfunction developed after a significant additional unexplained weight loss occurring after a long time period of stable weight after RYGB or OAGB. Total median \%EWL was $110.6 \%$ in all patients. In two thirds of patients, NAFLD was present already prior to surgery, but aggravated thereafter. In two patients, liver disease developed only after surgery.

In general, bariatric surgery has been successfully utilized to reverse or prevent further progression of NAFLD [18-22]. In a large meta-analysis, comprising 766 patients receiving paired liver biopsies after BPD, gastric banding, or RYGB, improvement or resolution of steatosis, steatohepatitis, and fibrosis was reported in 91.6, 81.3, and $65.5 \%$ of patients, respectively [3]. In contrast, although an improvement in steatosis and hepatic cell ballooning may be observed within the first year after these procedures, in a prospective study including 381 patients, 
although $95.7 \%$ maintained a fibrosis score $\leq \mathrm{F} 1$, fibrosis had progressed significantly after 5 years, and was associated with higher BMIs and increased insulin resistance [9]. However, apart from only slight impairment of liver histology, rarely, also severe deterioration leading to liver failure and death was described predominantly after JIB or BPD [12]. In contrast, there are only few single cases of significant liver dysfunction after laparoscopic RYGB [23]. To the best of our knowledge, there have been no reports of OAGB leading to a moderate or even excessive deterioration of liver function to the requirement of LT, as it was seen in our study.

The underlying mechanisms for liver deterioration after bariatric surgery have not yet been understood and prognostic surrogate parameters are lacking. It has been shown that purely restrictive procedures such as SG are feasible and effective also in patients with preexisting advanced liver disease [24]. To the best of our knowledge, severe liver deterioration after SG or gastric banding has not been described so far. In contrast, very recently, a randomized trial reported that patients with NASH undergoing RYGB were more susceptible to early transient deterioration of liver function compared to SG [25].

A negative impact on liver function after RYGB has been suggested to occur due to extended excluded limbs or distal versions of RYGB [26]. In our series, the dimensions of the common channels (median length $357.5 \mathrm{~cm}$ ) should have been more than sufficient for adequate absorption. Nevertheless, although BMI remained within a normal range, median \%EWL was significantly higher than average. It seems therefore apparent that an adequate limb length does not guarantee appropriate absorption in the individual patient. In fact, differing adaptation of the intestinal mucosa has been hypothesized [27].

Negative events after RYGB were furthermore reported to be largely associated with preoperatively diagnosed cirrhosis, alcohol abuse, and intraoperative complications [28]. The degree of liver disease, yet, had no effect on the perioperative outcome, but indicated an association with higher mortality in patients suffering from NASH at longterm follow-up [29, 30]. A systematic review in 108 mainly Child Pugh A patients undergoing bariatric surgery suggested an increased morbidity and mortality in these patients and described a decompensation of liver disease in six cases, as well as death due to fulminant hepatic failure in four cases [28]. This may lead to the question whether patients with a preexisting liver disease are more susceptible to a "second hit" due to rapid weight loss or undefined factors caused by malabsorptive procedures.

In our case series, both the facts that $60 \%$ of our patients showed preexisting liver disease and that a high total median $\%$ EWL of $110.6 .3 \%$ occurred may have contributed to patients' liver dysfunction. Importantly, the OAGB which is considered a safe procedure has led to a considerate liver impairment in five of our patients [31]. By elongation of the common limb or BP reversal, a significant improvement or even cessation of symptoms could be achieved in all patients after RYGB or OAGB.

Of note, up to $5 \%$ of patients with morbid obesity patients already suffer from undiagnosed cirrhosis at the time of bariatric surgery [2]. Symptoms can be relatively unspecific and even intraoperatively macroscopic liver disease may be easily overlooked [32]. In consequence, the presence of advanced liver disease should be thoroughly excluded prior to bariatric surgery. The importance of a meticulous postoperative followup including close monitoring of liver function tests needs to be emphasized. The focus might especially be brought on patients with previous morbid/super obesity, continuously impaired insulin resistance, and preoperative liver pathologies, as well as sudden and fast weight loss occurring independently from the primary procedure until prospective factors for postoperative liver deterioration have been established.

Large prospective clinical and basic research studies are required for a better understanding of the extent of our observation in this patient collective and for the determination of possible origins behind a deterioration of liver function after bariatric surgery.

Limitations Due to the retrospective nature of this study, we cannot give information on the overall prevalence of liver deterioration after bariatric surgery. Diagnostic methods were adapted to the clinical appearance of symptoms in each individual patient, and the prevalence of liver dysfunction might be underestimated.

\section{Conclusion}

To date, there are no recommendations for handling bariatric patients suffering from a certain degree of liver disease as there is insufficient evidence, so far. Considering the fact that obesity comes in hand with a high and increasing prevalence of NAFLD, guidelines for pre- and postoperative surveillance are warranted. We need to stress that in our cases, a very mild preoperative liver pathology has led to a clinically critical liver disease after the less malabsorptive procedures of OAGB and RYGB. In the presented cases, elongation of the resorption length of the bypass has led to an improvement in symptoms in all patients and this might be considered in patients showing a similar pattern of liver dysfunction after a bariatric procedure.

Acknowledgements Open access funding provided by Medical University of Vienna.

Author Contribution ME: data acquiration, drafting the article FBL: data review, revising the article for important intellectual content $\mathrm{AB}$ : histological analysis and stainings, revising the article for important intellectual content 
MT: revising the article for important intellectual content

GP: data review, revising the article for important intellectual content

KS: conception and design of the study, data review, drafting the article, and revising it critically for important intellectual content

\section{Compliance with Ethical Standards}

Conflict of Interest The authors declare that they have no conflict of interest.

Ethical Statement All procedures performed in this study were in accordance with the ethical standards of the institutional ethics committee and with the 1964 Helsinki declaration and its later amendments. Informed consent was obtained from all study participants where applicable.

Open Access This article is distributed under the terms of the Creative Commons Attribution 4.0 International License (http:// creativecommons.org/licenses/by/4.0/), which permits unrestricted use, distribution, and reproduction in any medium, provided you give appropriate credit to the original author(s) and the source, provide a link to the Creative Commons license, and indicate if changes were made.

\section{References}

1. Sanyal AJ. AGA technical review on nonalcoholic fatty liver disease. Gastroenterology. 2002;123(5):1705-25.

2. Sasaki A, Nitta H, Otsuka K, et al. Bariatric surgery and nonalcoholic fatty liver disease: current and potential future treatments. Front Endocrinol (Lausanne). 2014;5:164.

3. Hafeez $\mathrm{S}$, Ahmed MH. Bariatric surgery as potential treatment for nonalcoholic fatty liver disease: a future treatment by choice or by chance? J Obes. 2013;2013:839275.

4. Barker KB, Palekar NA, Bowers SP, et al. Non-alcoholic steatohepatitis: effect of Roux-en-Y gastric bypass surgery. Am J Gastroenterol. 2006;101(2):368-73.

5. Csendes A, Smok G, Burgos AM. Histological findings in the liver before and after gastric bypass. Obes Surg. 2006;16(5):607-11.

6. Moretto M, Kupski C, da Silva VD, et al. Effect of bariatric surgery on liver fibrosis. Obes Surg. 2012;22(7):1044-9.

7. Luyckx FH, Desaive C, Thiry A, et al. Liver abnormalities in severely obese subjects: effect of drastic weight loss after gastroplasty. Int J Obes Relat Metab Disord. 1998;22(3):222-6.

8. Stratopoulos C, Papakonstantinou A, Terzis I, et al. Changes in liver histology accompanying massive weight loss after gastroplasty for morbid obesity. Obes Surg. 2005;15(8):1154-60.

9. Mathurin P, Hollebecque A, Arnalsteen L, et al. Prospective study of the long-term effects of bariatric surgery on liver injury in patients without advanced disease. Gastroenterology. 2009;137(2): 532-40.

10. Kral JG, Thung SN, Biron S, et al. Effects of surgical treatment of the metabolic syndrome on liver fibrosis and cirrhosis. Surgery. 2004;135(1):48-58.

11. Baltasar A, Serra C, Perez N, et al. Clinical hepatic impairment after the duodenal switch. Obes Surg. 2004;14(1):77-83.

12. Geerts A, Darius T, Chapelle T, et al. The multicenter Belgian survey on liver transplantation for hepatocellular failure after bariatric surgery. Transplant Proc. 2010;42(10):4395-8.

13. Requarth JA, Burchard KW, Colacchio TA, et al. Long-term morbidity following jejunoileal bypass. The continuing potential need for surgical reversal. Arch Surg. 1995;130(3):318-25.
14. Torgersen Z, Osmolak A, Forse RA. Sleeve gastrectomy and Roux En Y gastric bypass: current state of metabolic surgery. Curr Opin Endocrinol Diabetes Obes. 2014;21(5):352-7.

15. Lee WJ, Yu PJ, Wang W, et al. Laparoscopic Roux-en-Y versus mini-gastric bypass for the treatment of morbid obesity: a prospective randomized controlled clinical trial. Ann Surg. 2005;242(1): 20-8.

16. van de Laar A. Bariatric outcomes longitudinal database (BOLD) suggests excess weight loss and excess BMI loss to be inappropriate outcome measures, demonstrating better alternatives. Obes Surg. 2012;22(12):1843-7.

17. WHO. Report of a WHO consultation on obesity. Obesity: preventing and managing the global epidemic 2000;894.

18. Furuya CK, Jr., de Oliveira CP, de Mello ES, Faintuch J, Raskovski A, Matsuda M, et al. Effects of bariatric surgery on nonalcoholic fatty liver disease: preliminary findings after 2 years. J Gastroenterol Hepatol 2007;22(4):510-514. PubMed PMID: 17376042

19. Liu X, Lazenby AJ, Clements RH, et al. Resolution of nonalcoholic steatohepatits after gastric bypass surgery. Obes Surg. 2007;17(4): 486-92.

20. Sjostrom L, Narbro K, Sjostrom CD, et al. Effects of bariatric surgery on mortality in Swedish obese subjects. N Engl J Med. 2007;357(8):741-52.

21. Clark JM, Alkhuraishi AR, Solga SF, Alli P, Diehl AM, Magnuson TH. Roux-en-Y gastric bypass improves liver histology in patients with non-alcoholic fatty liver disease. Obes Res 2005 Jul;13(7): 1180-1186. PubMed

22. Shimizu H, Phuong V, Maia M, et al. Bariatric surgery in patients with liver cirrhosis. Surg Obes Relat Dis. 2013;9(1):1-6.

23. Mahawar KK, Parmar C, Graham Y, et al. Monitoring of liver function tests after Roux-en-Y gastric bypass: an examination of evidence base. Obes Surg. 2016;26(10):2516-22.

24. Lazzati A, Iannelli A, Schneck AS, et al. Bariatric surgery and liver transplantation: a systematic review a new frontier for bariatric surgery. Obes Surg. 2015;25(1):134-42.

25. Kalinowski P, Paluszkiewicz R, Ziarkiewicz-Wroblewska B, Wroblewski T, Remiszewski P, Grodzicki M, et al. Liver function in patients with nonalcoholic fatty liver disease randomized to Roux-en-Y gastric bypass versus sleeve gastrectomy: a secondary analysis of a randomized clinical trial. Ann Surg 2017.

26. Sugerman HJ, Kellum JM, DeMaria EJ. Conversion of proximal to distal gastric bypass for failed gastric bypass for superobesity. J Gastrointest Surg. 1997;1(6):517-24. discussion 24-6

27. Shaw D, Gohil K, Basson MD. Intestinal mucosal atrophy and adaptation. World J Gastroenterol. 2012 Nov 28;18(44):6357-75.

28. Jan A, Narwaria M, Mahawar KK. A systematic review of bariatric surgery in patients with liver cirrhosis. Obes Surg. 2015;25(8): 1518-26.

29. Wolter S, Dupree A, Coelius C, El Gammal A, Kluwe J, Sauer N, et al. Influence of liver disease on perioperative outcome after bariatric surgery in a northern German cohort. Obes Surg. 2016.

30. Goossens N, Hoshida Y, Song WM, et al. Nonalcoholic steatohepatitis is associated with increased mortality in obese patients undergoing bariatric surgery. Clin Gastroenterol Hepatol. 2016;14(11):1619-28.

31. Garcia-Caballero M, Carbajo M. One anastomosis gastric bypass: a simple, safe and efficient surgical procedure for treating morbid obesity. Nutr Hosp 2004;19(6):372-375. PubMed

32. Teixeira AR, Bellodi-Privato M, Carvalheira JB, et al. The incapacity of the surgeon to identify NASH in bariatric surgery makes biopsy mandatory. Obes Surg. 2009;19(12):1678-84. 